\title{
Kinetics and mechanism of oxidation of aliphatic alcohols by tetrabutylammonium tribromide
}

\author{
MANJU BAGHMAR and PRADEEP K SHARMA* \\ Department of Chemistry, JNV University, Jodhpur 342 005, India \\ e-mail: pradeep2sharma@usa.net
}

MS received 20 May 2000; revised 3 January 2001

\begin{abstract}
Oxidation of nine primary aliphatic alcohols by tetrabutylammonium tribromide (TBATB) in aqueous acetic acid leads to the formation of the corresponding aldehydes. The reaction is first order with respect to TBATB. Michaelis-Menten type kinetics is observed with respect to alcohols. The reaction failed to induce the polymerization of acrylonitrile. Tetrabutylammonium chloride has no effect on the reaction rate. The proposed reactive oxidizing species is the tribromide ion. The oxidation of $\left[1,1-{ }^{2} \mathrm{H}_{2}\right]$ ethanol exhibits a substantial kinetic isotope effect. The effect of solvent composition indicates that the rate increases with increase in the polarity of the solvent. The reaction is susceptible to both polar and steric effects of substituents. A mechanism involving transfer of a hydride ion in the ratedetermining step has been proposed.
\end{abstract}

Keywords. Aliphatic alcohols; hydride ion transfer; tetrabutylammonium tribromide.

\section{Introduction}

Tetraalkylammonium polyhalides are widely used as halogenating reagents in synthetic organic chemistry ${ }^{1-3}$. Recently, tetrabutylammonium tribromide (TBATB) has been used for bromination of some selected organic substrates ${ }^{4}$. There are, however, only a few reports regarding their use as oxidizing and brominating agents in synthetic chemistry ${ }^{5-7}$. These compounds are more suitable than molecular halogens because they are solids and hence easy to handle, and owing to their stability, selectivity and excellent product yields. We have been interested in the kinetic and mechanistic studies of the reactions of polyhalides and have already reported some of them ${ }^{8-10}$. We discuss here the kinetics of oxidation of nine aliphatic alcohols by TBATB in aqueous acetic acid solution. Attempts have been made to correlate rate and structure in this reaction. A suitable mechanism has also been proposed.

\section{Experimental}

\subsection{Materials}

All the alcohols used were of commercial grade. Methanol, ethanol, and 1-propanol were purified by the magnesium alkoxide method ${ }^{11}$. Other alcohols were dried over anhydrous

*For correspondence 
potassium carbonate and fractionated. TBATB was prepared by the reported method ${ }^{1}$ and its purity checked by the iodometric method. $\left[1,1-{ }^{2} \mathrm{H}_{2}\right]$ Ethanol was prepared by reported methods ${ }^{12}$. Its isotopic purity, ascertained by its ${ }^{1} \mathrm{H}$ NMR spectrum, was $94 \pm 4 \%$. Acetic acid was refluxed with chromic oxide and acetic anhydride for $6 \mathrm{~h}$ and then fractionally distilled. All other reagents were commercial products and were purified by the usual methods ${ }^{13}$.

\subsection{Product analysis}

Product analysis was carried out under kinetic conditions. In a typical experiment, ethanol (4.6 g, 0.1 mol), $\mathrm{KBr}(0.2 \mathrm{~mol})$ and TBATB (4.82 $\mathrm{g}, 0.01 \mathrm{~mol})$ were made up to $50 \mathrm{ml}$ in 1:1 $(v / v)$ aqueous acetic acid and kept in the dark for $\approx 10 \mathrm{~h}$ to ensure completion of the reaction. The solution was then treated with an excess $(200 \mathrm{ml})$ of a saturated solution of 2,4-dinitrophenylhydrazine in $2 \mathrm{~mol} \mathrm{dm}^{-3} \mathrm{HCl}$ and kept overnight under refrigeration. The precipitated 2,4-dinitrophenylhydrazone (DNP) was filtered off, dried, weighed, recrystallized from ethanol, and weighed again. Yields of DNP before and after recrystallization were $4.31 \mathrm{~g}(94 \%)$ and $3.72 \mathrm{~g}(81 \%)$ respectively, based on the consumption of TBATB. The DNP was found to be identical (m.p. and mixed m.p.) with the DNP of acetaldehyde. In similar experiments with other alcohols, yields of the DNPs were in the range of $77-87 \%$ after recrystallization.

\subsection{Kinetic measurements}

The reactions were studied under pseudo-first-order conditions by using an excess $(\times 15$ or greater) of the substrate over TBATB. The solvent was 1:1 $(v / v)$ acetic acid-water, unless mentioned otherwise. Tribromide ion is known to dissociate to a large extent with bromine and bromide ion. The value ${ }^{14}$ of the dissociation constant in $1: 1(\mathrm{v} / \mathrm{v})$ acetic acid-water is $\approx 0.02 \mathrm{~mol} \mathrm{dm}^{-3}$. To suppress the dissociation, all kinetic runs were carried out in the presence of an excess $\left(0.2 \mathrm{~mol} \mathrm{dm}^{-3}\right)$ of potassium bromide. The reactions were studied at constant temperature $( \pm 0 \cdot 1 \mathrm{~K})$ and were followed by monitoring the decrease in the concentration of TBATB at $394 \mathrm{~nm}$ for up to $80 \%$ reaction. Pseudo-first-order rate constants, $k_{\text {obs }}$, were evaluated from linear plots $(r>0.995)$ of $\log$ [TBATB] against time. Duplicate kinetic runs showed that the rate constants are reproducible to within $\pm 3 \%$. Simple and multivariate regression analyses were carried out by the least-squares method.

\section{Results}

\subsection{Stoichiometry}

The oxidation of alcohols by TBATB results in the formation of corresponding aldehydes. The overall reaction may be represented as,

$$
\mathrm{RCH}_{2} \mathrm{OH}+\left(\mathrm{C}_{4} \mathrm{H}_{9}\right) \mathrm{N}^{+} \mathrm{Br}_{3}^{-} \rightarrow \mathrm{RCHO}+\left(\mathrm{C}_{4} \mathrm{H}_{9}\right) \mathrm{N}^{+} \mathrm{Br}^{-}+2 \mathrm{HBr} .
$$

\subsection{Rate laws}

The reactions are of first order with respect to TBATB. Further, the pseudo-first-order rate constant, $k_{\mathrm{obs}}$, are independent of the initial concentration of TBATB. The reaction 
rate increases with an increase in the concentration of the alcohol but not linearly (table 1 ). A plot of $1 / k_{\text {obs }}$ against $1 /[$ alcohol] is linear $(r>0.995)$ with an intercept on the rateordinate. Thus, Michaelis-Menten type kinetics is observed with respect to the alcohols. This leads to the postulation of the following overall mechanism ((2) and (3)) and rate law, (4)

$$
\begin{aligned}
& \text { Alcohol + oxidant } \square \text { [complex }], \\
& {[\text { complex }] \stackrel{k_{2}}{\longrightarrow} \text { products, }} \\
& \text { Rate }=k_{2} K[\text { alcohol] [oxidant }] /(1+K[\text { alcohol }]) .
\end{aligned}
$$

Dependence of reaction rate on reductant concentration was studied at different temperatures and the values of $K$ and $k_{2}$ were evaluated from the double reciprocal plots. Thermodynamic parameters of complex formation and activation parameters of decomposition of the complexes were calculated from the values of $K$ and $k_{2}$ respectively at different temperatures (tables 2 and 3).

\subsection{Induced polymerization of acrylonitrile}

Oxidation of ethanol, in an atmosphere of nitrogen, failed to induce polymerization of acrylonitrile. Further, the addition of acrylonitrile had no effect on the rate of oxidation (table 1).

\subsection{Kinetic isotope effect}

To ascertain the importance of the cleavage of the $\alpha-\mathrm{C}-\mathrm{H}$ bond in the rate-determining step, the oxidation of $\left[1,1-{ }^{2} \mathrm{H}_{2}\right]$ ethanol was studied. Results showed the presence of a primary kinetic isotope effect (table 3 ). The rate of oxidation of the deuteriated alcohol was corrected for the protio alcohol present.

Table 1. Rate constants for the oxidation of ethanol by TBATB at $288 \mathrm{~K}$.

\begin{tabular}{lcc}
\hline $10^{3}[\mathrm{TBATB}]\left(\mathrm{mol} \mathrm{dm}^{-3}\right)$ & [Alcohol] $\left(\mathrm{mol} \mathrm{dm}^{-3}\right)$ & $10^{5} k_{\mathrm{obs}}\left(\mathrm{s}^{-1}\right)$ \\
\hline $1 \cdot 0$ & $0 \cdot 10$ & $20 \cdot 5$ \\
$1 \cdot 0$ & $0 \cdot 20$ & $31 \cdot 2$ \\
$1 \cdot 0$ & $0 \cdot 40$ & $42 \cdot 3$ \\
$1 \cdot 0$ & $0 \cdot 60$ & $48 \cdot 0$ \\
$1 \cdot 0$ & $0 \cdot 80$ & $51 \cdot 5$ \\
$1 \cdot 0$ & $1 \cdot 00$ & $53 \cdot 8$ \\
$1 \cdot 0$ & $1 \cdot 50$ & $57 \cdot 3$ \\
$1 \cdot 0$ & $3 \cdot 00$ & $61 \cdot 2$ \\
$2 \cdot 0$ & $0 \cdot 20$ & $31 \cdot 0$ \\
$4 \cdot 0$ & $0 \cdot 20$ & $31 \cdot 8$ \\
$6 \cdot 0$ & $0 \cdot 20$ & $32 \cdot 1$ \\
$8 \cdot 0$ & $0 \cdot 20$ & $31 \cdot 5$ \\
$1 \cdot 0$ & $0 \cdot 20$ & $31 \cdot 6 *$ \\
\hline
\end{tabular}

*Contained 0.005 $\mathrm{mol} \mathrm{dm}^{-3}$ acrylonitrile 
Table 2. Formation constants of the TBATB- $\mathrm{RCH}_{2} \mathrm{OH}$ complexes and thermodynamic parameters.

\begin{tabular}{|c|c|c|c|c|c|c|c|}
\hline \multirow[b]{2}{*}{ Alcohol } & \multicolumn{4}{|c|}{$K\left(\mathrm{dm}^{3} \mathrm{~mol}^{-1}\right)$ at } & \multirow{2}{*}{$\begin{array}{c}\Delta H \\
\left(\mathrm{~kJ} \mathrm{~mol}^{-1}\right)\end{array}$} & \multirow{2}{*}{$\begin{array}{c}\Delta S \\
\left(\mathrm{~J} \mathrm{~mol}^{-1} \mathrm{~K}^{-1}\right)\end{array}$} & \multirow{2}{*}{$\begin{array}{c}\Delta G \\
\left(\mathrm{~kJ} \mathrm{~mol}^{-1}\right)\end{array}$} \\
\hline & $288 \mathrm{~K}$ & $298 \mathrm{~K}$ & $308 \mathrm{~K}$ & $318 \mathrm{~K}$ & & & \\
\hline $\mathrm{H}$ & $4 \cdot 81$ & $4 \cdot 14$ & $3 \cdot 40$ & $2 \cdot 72$ & $-16 \cdot 9 \pm 0 \cdot 8$ & $-37 \pm 3$ & $-5.93 \pm 0.6$ \\
\hline $\mathrm{Me}$ & $4 \cdot 52$ & $3 \cdot 80$ & $3 \cdot 32$ & $2 \cdot 63$ & $-16 \cdot 0 \pm 0 \cdot 8$ & $-34 \pm 3$ & $-5 \cdot 82 \pm 0 \cdot 7$ \\
\hline $\mathrm{Et}$ & $5 \cdot 15$ & 4.46 & $3 \cdot 82$ & $3 \cdot 11$ & $-15 \cdot 5 \pm 0.7$ & $-31 \pm 2$ & $-6 \cdot 16 \pm 0 \cdot 5$ \\
\hline$n$-Pr & $4 \cdot 50$ & $3 \cdot 61$ & $2 \cdot 89$ & $2 \cdot 41$ & $-18 \cdot 5 \pm 0 \cdot 1$ & $-43 \pm 1$ & $-5 \cdot 67 \pm 0 \cdot 1$ \\
\hline$n-\mathrm{Bu}$ & $5 \cdot 10$ & $4 \cdot 51$ & $3 \cdot 72$ & $3 \cdot 02$ & $-15 \cdot 9 \pm 0 \cdot 9$ & $-33 \pm 3$ & $-6 \cdot 14 \pm 0 \cdot 7$ \\
\hline$i-\operatorname{Pr}$ & $5 \cdot 33$ & $4 \cdot 60$ & $3 \cdot 80$ & $3 \cdot 00$ & $-17 \cdot 0 \pm 0 \cdot 9$ & $-37 \pm 3$ & $-6 \cdot 21 \pm 0 \cdot 7$ \\
\hline $\mathrm{ClCH}_{2}$ & $4 \cdot 88$ & $4 \cdot 23$ & $3 \cdot 55$ & $2 \cdot 85$ & $-16 \cdot 1 \pm 0 \cdot 8$ & $-34 \pm 3$ & $-6 \cdot 01 \pm 0 \cdot 6$ \\
\hline $\mathrm{MeOCH}_{2}$ & $5 \cdot 63$ & 4.92 & $4 \cdot 25$ & $3 \cdot 41$ & $-15 \cdot 0 \pm 0 \cdot 9$ & $-29 \pm 3$ & $-6 \cdot 40 \pm 0 \cdot 7$ \\
\hline$t-\mathrm{Bu}$ & $5 \cdot 25$ & $4 \cdot 55$ & $3 \cdot 83$ & $3 \cdot 06$ & $-16 \cdot 1 \pm 0 \cdot 8$ & $-34 \pm 3$ & $-6 \cdot 19 \pm 0 \cdot 7$ \\
\hline $\mathrm{MeCD}_{2}$ & $4 \cdot 60$ & $3 \cdot 82$ & $3 \cdot 10$ & $2 \cdot 50$ & $-17 \cdot 9 \pm 0 \cdot 5$ & $-41 \pm 2$ & $-5 \cdot 78 \pm 0.7$ \\
\hline
\end{tabular}

Table 3. Rate constants for the decomposition of TBATB- $\mathrm{RCH}_{2} \mathrm{OH}$ complexes and the activation parameters.

\begin{tabular}{|c|c|c|c|c|c|c|c|}
\hline \multirow[b]{2}{*}{ Alcohol } & \multicolumn{4}{|c|}{$10^{5} k_{2}\left(\mathrm{dm}^{3} \mathrm{~mol}^{-1} \mathrm{~s}^{-1}\right)$ at } & \multirow{2}{*}{$\begin{array}{c}\Delta H^{\#} \\
\left(\mathrm{~kJ} \mathrm{~mol}^{-1}\right)\end{array}$} & \multirow{2}{*}{$\begin{array}{c}\Delta S^{\#} \\
\left(\mathrm{~J} \mathrm{~mol}^{-1} \mathrm{~K}^{-1}\right)\end{array}$} & \multirow{2}{*}{$\begin{array}{c}\Delta G^{\#} \\
\left(\mathrm{~kJ} \mathrm{~mol}^{-1}\right)\end{array}$} \\
\hline & $288 \mathrm{~K}$ & $298 \mathrm{~K}$ & $308 \mathrm{~K}$ & $318 \mathrm{~K}$ & & & \\
\hline $\mathrm{H}$ & $1 \cdot 31$ & $3 \cdot 81$ & $11 \cdot 2$ & $31 \cdot 3$ & $78 \cdot 1 \pm 0 \cdot 8$ & $-68 \pm 3$ & $98 \cdot 1 \pm 0 \cdot 6$ \\
\hline $\mathrm{Me}$ & $65 \cdot 7$ & 138 & 288 & 612 & $57 \cdot 4 \pm 0 \cdot 6$ & $-108 \pm 2$ & $89 \cdot 4 \pm 0 \cdot 5$ \\
\hline Et & 108 & 220 & 430 & 880 & $50 \cdot 5 \pm 0.8$ & $-127 \pm 3$ & $88 \cdot 2 \pm 0 \cdot 7$ \\
\hline$n-\operatorname{Pr}$ & 178 & 350 & 672 & 1310 & $48 \cdot 0 \pm 0 \cdot 6$ & $-131 \pm 3$ & $87 \cdot 0 \pm 0 \cdot 5$ \\
\hline$n-\mathrm{Bu}$ & 207 & 397 & 735 & 1390 & $46 \cdot 2 \pm 0 \cdot 4$ & $-136 \pm 1$ & $86 \cdot 7 \pm 0 \cdot 3$ \\
\hline$i-\operatorname{Pr}$ & 280 & 555 & 1020 & 1810 & $44 \cdot 7 \pm 0 \cdot 2$ & $-139 \pm 1$ & $86 \cdot 0 \pm 0 \cdot 2$ \\
\hline $\mathrm{ClCH}_{2}$ & 1.91 & $5 \cdot 04$ & $13 \cdot 1$ & $33 \cdot 2$ & $69 \cdot 9 \pm 0.7$ & $-93 \pm 2$ & $97 \cdot 5 \pm 0 \cdot 5$ \\
\hline $\mathrm{MeOCH}_{2}$ & $12 \cdot 8$ & $29 \cdot 9$ & $68 \cdot 7$ & 154 & $60 \cdot 6 \pm 0 \cdot 6$ & $-109 \pm 2$ & $93 \cdot 1 \pm 0 \cdot 4$ \\
\hline$t-\mathrm{Bu}$ & 2650 & 4100 & 6010 & 8940 & $28 \cdot 2 \pm 0 \cdot 3$ & $-178 \pm 1$ & $81 \cdot 0 \pm 0 \cdot 2$ \\
\hline $\mathrm{MeCD}_{2}$ & $20 \cdot 9$ & $44 \cdot 8$ & $92 \cdot 3$ & 197 & $54 \cdot 2 \pm 0 \cdot 8$ & $-128 \pm 3$ & $92 \cdot 1 \pm 0 \cdot 6$ \\
\hline$k_{\mathrm{H}} / k_{\mathrm{D}}$ & $3 \cdot 14$ & 3.08 & $3 \cdot 12$ & $3 \cdot 10$ & & & \\
\hline
\end{tabular}

\subsection{Effect of tetrabutylammonium chloride}

Addition of tetrabutylammonium chloride (TBACl) had no effect on the rates of oxidation (table 4).

\subsection{Effect of solvent composition}

Rate of oxidation was determined in solvents containing different amounts of acetic acid and water. It was observed that rates increase with increase in the amount of water in the solvent mixture. To determine whether the solvent composition affects the formation constant, $K$, and/or rate constant, $k_{2}$, the variation of rate with concentration of ethanol was studied in solutions of different compositions. The results (table 5) showed that the 
Table 4. Effect of tetrabutylammonium chloride on the rate of oxidation of ethanol by TBATB.

\begin{tabular}{|c|c|c|c|c|c|c|}
\hline$[$ TBATB $]=0.001 \mathrm{~mol} \mathrm{dm}^{-}$ & [eth & $=1 \cdot 0$ & ol dm ${ }^{-3}$ & Temp & $288 \mathrm{~K}$ & \\
\hline $10^{3}[\mathrm{TBACl}]\left(\mathrm{mol} \mathrm{dm}^{-3}\right)$ & $0 \cdot 0$ & 0.5 & 1.0 & $2 \cdot 0$ & $2 \cdot 0$ & $4 \cdot 0$ \\
\hline $10^{5} k_{\text {obs }}\left(\mathrm{s}^{-1}\right)$ & $53 \cdot 8$ & $52 \cdot 8$ & $53 \cdot 1$ & $52 \cdot 6$ & $53 \cdot 3$ & $52 \cdot 0$ \\
\hline
\end{tabular}

Table 5. Dependence of rates for alcohol oxidation by TBATB on substrate concentration in solvents of different solvent compositions.

\begin{tabular}{|c|c|c|c|c|c|}
\hline \multirow{2}{*}{$\begin{array}{l}\text { Ethanol } \\
\left(\mathrm{mol} \mathrm{dm}^{-3}\right)\end{array}$} & \multicolumn{5}{|c|}{$10^{4} k_{\mathrm{obs}}\left(\mathrm{s}^{-1}\right)[$ at $\% \operatorname{AcOH}(v / v)]$} \\
\hline & 25 & 40 & 50 & 60 & 72 \\
\hline $0 \cdot 1$ & $9 \cdot 72$ & $4 \cdot 45$ & $2 \cdot 05$ & 1.09 & $0 \cdot 40$ \\
\hline $0 \cdot 2$ & $15 \cdot 0$ & $6 \cdot 60$ & $3 \cdot 12$ & 1.63 & 0.59 \\
\hline $0 \cdot 4$ & $20 \cdot 7$ & $8 \cdot 71$ & $4 \cdot 23$ & $2 \cdot 18$ & 0.79 \\
\hline $0 \cdot 6$ & $23 \cdot 6$ & 9.75 & $4 \cdot 80$ & $2 \cdot 45$ & 0.88 \\
\hline $0 \cdot 8$ & $25 \cdot 5$ & $10 \cdot 4$ & $5 \cdot 15$ & $2 \cdot 62$ & 0.94 \\
\hline $1 \cdot 0$ & $26 \cdot 7$ & $10 \cdot 8$ & $5 \cdot 38$ & $2 \cdot 73$ & 0.98 \\
\hline $1 \cdot 5$ & $28 \cdot 5$ & $11 \cdot 4$ & 5.73 & $2 \cdot 89$ & $1 \cdot 04$ \\
\hline $3 \cdot 0$ & $30 \cdot 6$ & $12 \cdot 0$ & $6 \cdot 12$ & $3 \cdot 07$ & $1 \cdot 10$ \\
\hline$K\left(\mathrm{dm}^{3} \mathrm{~mol}^{-3}\right)$ & $4 \cdot 16$ & $5 \cdot 33$ & 4.52 & 4.95 & $5 \cdot 15$ \\
\hline $10^{4} k_{2}\left(\mathrm{~s}^{-1}\right)$ & $33 \cdot 1$ & 12.8 & $6 \cdot 57$ & $3 \cdot 28$ & $1 \cdot 17$ \\
\hline
\end{tabular}

rate constants, $k_{2}$, vary considerably while the formation constant, $K$, remains practically independent of solvent composition.

\section{Discussion}

There is no significant isokinetic relationship between activation entropy and enthalpy of the oxidation of nine primary alcohols $\left(r^{2}=0.5568\right)$. Correlation between the calculated values of enthalpies and entropies of activation is often vitiated by the experimental errors associated with them. Exner ${ }^{15}$ has suggested an alternative method of testing the validity of the isokinetic relationship. An Exner plot between $\log k_{2}$ at 288 and $318 \mathrm{~K}$ was linear $\left(r^{2}=0.9982\right.$; slope $\left.0.7675 \pm 0.0122\right)$. The value of isokinetic temperature evaluated from this plot is $485 \pm 17 \mathrm{~K}$. The linear isokinetic correlation suggests that all the alcohols are oxidized by the same mechanism and changes in the rates are governed by changes in both the enthalpy and entropy of activation.

We have carried out some conductivity measurements to determine the nature of TBATB in aqueous acetic acid solutions. It is observed that acetic acid has very low conductivity. Addition of TBATB increases the conductivity of acetic acid. We measured the conductivity of TBATB in solvents containing different proportions of acetic acid $(100-30 \%)$ and water. We found that conductivity increases sharply as the water content is initially increased but reaches a limiting value in about $60 \%$ acetic acid-water mixture. 
Therefore, TBATB can be considered an ionic compound, which exists under our reaction conditions as tetrabutylammonium and tribromide ions, (5) below. Absence of any effect due to added tetrabutylammonium ion also indicates that the equilibrium (5) lies far towards the right,

$$
\left(\mathrm{C}_{4} \mathrm{H}_{9}\right)_{4} \mathrm{NBr}_{3} \square\left(\mathrm{C}_{4} \mathrm{H}_{9}\right)_{4} \mathrm{~N}^{+}+\mathrm{Br}_{3}^{-} .
$$

Tribromide ion is known to dissociate to bromine and bromide ion and dissociation constant values have been reported ${ }^{14}$. However, in the presence of large excess of bromide ion, the following reaction is suppressed. Thus in the present reaction the reactive oxidising species is the tribromide ion,

$$
\mathrm{Br}_{3}^{-} \square \mathrm{Br}_{2}+\mathrm{Br}^{-} \text {. }
$$

The oxidation of alcohols by bromine is reported to be much faster ${ }^{16}$ as compared to this reaction. This also supports the view that bromine is not the reactive species in this oxidation.

\subsection{Solvent composition effect}

Increase in the rate constant of disproportionation of the intermediate complex with an increase in the polarity of the medium suggests that the transition state is more polar than the reactants. The plot of $\log k_{2}$ against the inverse of the dielectric constant is nonlinear. The solvent effect was analysed using Grunwald-Winstein equation ${ }^{17}$,

$$
\log k_{2}=\log k_{0}+m Y \text {. }
$$

The plot of $\log k_{2}$ versus $Y$ is linear $(r=0.9997)$ with $m=0.78 \pm 0.01$. The value of $m$ suggests a transition state which is more polar than the reactants. Thus considerable charge separation takes place in the transition state of the reaction.

\subsection{Correlation analysis of reactivity}

The data in table 2 show that the formation constants of alcohol-TBATB complexes are not sensitive to the nature of the substituent in the alcohol molecule. The rate constants, $k_{2}$ of disproportionation of the complexes (table 3), however, showed considerable variation.

The rate constants, $k_{2}$, of oxidation of alcohols failed to yield any significant correlation separately with Taft's ${ }^{18} \alpha^{*}$ and $E_{s}$. The correlation with $\sigma^{*}$ accounts for $\approx 82 \%$ of the data but correlation is not acceptable by Exner's criterion ${ }^{19}$,

$$
\begin{aligned}
\log k_{2} & =-2 \cdot 16( \pm 0.39) \Sigma \sigma^{*}-2 \cdot 95, \\
r^{2} & =0.8167, \mathrm{sd}=0 \cdot 49, n=9, \psi=0.33, \mathrm{~T}=288 \mathrm{~K}, \\
\log k_{2} & =-1 \cdot 20( \pm 0.34) \Sigma E_{s}-3 \cdot 51 \\
r^{2} & =0.6357, \mathrm{sd}=0.69, n=9, \psi=0.48, \mathrm{~T}=288 \mathrm{~K} .
\end{aligned}
$$

Here $n$ is the number of data points and $\psi$ is Exner's statistical parameter ${ }^{19}$. The rate constants were, therefore, correlated in terms of Pavelich-Taft's ${ }^{20}$ dual substituentparameter as below, 


$$
\log k=\rho^{*} \Sigma \sigma^{*}+\delta \Sigma E_{s}+\log k_{0}
$$

The values of the substituent constants were obtained from the compilation by Wiberg ${ }^{18}$. the correlations are excellent, reaction constants being negative (table 6). There is no significant collinearity $\left(r^{2}=0 \cdot 2322\right)$ between $\sigma^{*}$ and $E_{s}$ of the nine substituents.

\section{Mechanism}

Negative polar reaction constant indicates an electron-deficient carbon centre in the transition state of the rate-determining step. Negative steric reaction constants point to steric acceleration of the reaction. This may be explained on the basis of the high ground state energy of the sterically crowded alcohols. Since the crowding is relieved in the product aldehyde as well as in the transition state leading to it, the transition state energy of the crowded and uncrowded alcohols do not differ much and steric acceleration, therefore, results.

From the rate law, it is apparent that an intermediate complex is formed in a rapid preequilibrium. However, with the present data, it is not possible to conclude definitely about the nature of the complex. A complex may be formed by interaction between the non-bonded pairs of electrons of the alcohol-oxygen and tribromide ion. The formation of a similar complex has been postulated in the oxidation of the alcohols ${ }^{21}$ by pyridinium hydrobromide perbromide (PHPB) also. Formation of moderately stable complexes is also supported by the values of the thermodynamic parameters. Complex formation is favoured by the enthalpy term but there is loss of entropy indicating the formation of a rigid structure.

Temperature invariance of the primary kinetic isotope effect (cf. table 3) can be interpreted in terms of a mechanism in which two bonds are cleaved more or less synchronously. Therefore, a rate-determining step involving cleavage of both the $\mathrm{C}-\mathrm{H}$ and $\mathrm{O}-\mathrm{H}$ bonds can be envisaged. The low magnitude of the polar reaction constants also supports the occurrence of a synchronous mechanism. However, correlation analysis of the substituent effect indicated the presence of an electron-deficient carbon centre in the transition state. It seems, therefore, that in the transition state the cleavage of the $\mathrm{C}-\mathrm{H}$ bond, yielding an electron-deficient carbon centre, is before the cleavage of the $\mathrm{O}-\mathrm{H}$ bond. The transition state remains polar in this mechanism and is consistent with the observed solvent effect. The solvent effect is similar to that found for synchronous homopolar reactions. A nonlinear transition state, implied in the synchronous mechanism, is supported by the relatively low magnitude of the kinetic isotope effect $\left(k_{\mathrm{H}} / k_{\mathrm{D}} \approx 3 \cdot 1\right)$. The oxidation of benzyl alcohol by $\mathrm{PHPB}^{21}$ also was reported to involve a nonlinear transition state and the kinetic isotope effect was found to be $\approx 3 \cdot 5$. The mechanism depicted in scheme 1 accounts for all the observed results.

Table 6. Temperature dependence of the reaction constants.

\begin{tabular}{lccccc}
\hline Temp. (K) & $\rho^{*}$ & $\delta$ & $R^{2}$ & $\mathrm{sd}$ & $\psi$ \\
\hline 288 & $-1.62 \pm 0.008$ & $-0.73 \pm 0.005$ & 0.9999 & 0.009 & 0.008 \\
298 & $-1.52 \pm 0.004$ & $-0.66 \pm 0.003$ & 0.9998 & 0.005 & 0.011 \\
308 & $-1.41 \pm 0.004$ & $-0.58 \pm 0.003$ & 0.9999 & 0.005 & 0.008 \\
318 & $-1.31 \pm 0.006$ & $-0.51 \pm 0.004$ & 0.9997 & 0.007 & 0.014 \\
\hline
\end{tabular}




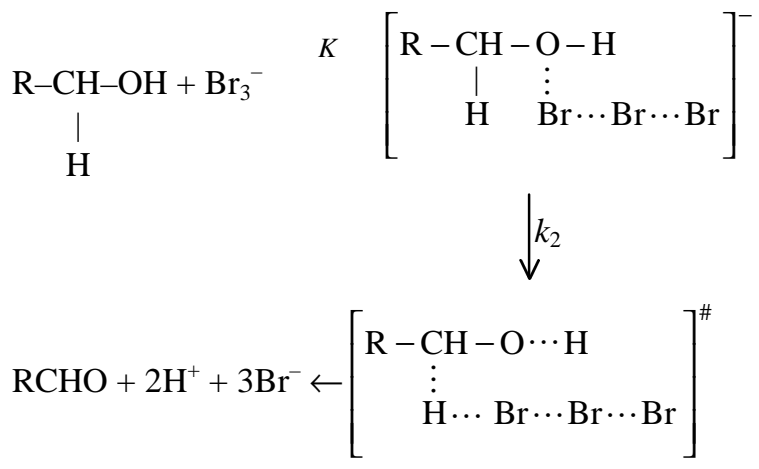

Scheme 1.

The observed negative entropy of activation also supports the mechanism. As the charge separation takes place, the charged ends become highly solvated. This results in immobilization of a large number of solvent molecules, reflected by the loss of entropy ${ }^{22}$.

\section{Acknowledgements}

Thanks are due to the Council of Scientific and Industrial Research, New Delhi for financial assistance and to Prof. Kalyan K Banerji for his valuable help and suggestions.

\section{References}

1. Kajigaeshi S, Kakinami T, Okamoto T and Fujisaki S 1987 Bull. Chem. Soc. Jpn. 601159

2. Kajigaeshi S, Kakinami T, Yamasaki H, Fujisaki S and Okamoto T 1988 Bull. Chem. Soc. Jpn. 612681

3. Buckles R E, Popov A I, Zelenzy W F and Smith R J 1951 J. Am. Chem. Soc. 734525

4. Choudhary M K, Khan A T and Patel B K 1998 Tetrahedron Lett. 398163

5. Berthalot J, Guette C, Ouchefoune M, Desbens P L and Basselier J J 1986 J. Chem. Res. (S) 381; 1986 Synth. Commun. 161641

6. Kajigaeshi S, Kawamuki H and Fujisaki S 1989 Bull. Chem. Soc. Jpn. 622585

7. Kajigaeshi S, Morikawa Y, Fujisaki S, Kakinami T and Nishihira K 1991 Bull. Chem. Soc. Jpn. 64336

8. Suri D, Kothari S and Banjerji K K 1996 Int. J. Chem. Kinet. 28681

9. Rao P S C, Goel S, Kothari S and Banjerji K K 1998 Indian J Chem B37 1129

10. Bohra A, Sharma P K and Banerji K K 1990 J. Chem. Res. (S) 308; (M) 1343

11. Vogel A I 1967 A text book of organic chemistry (London: Longmans) p. 167

12. Kaplan L 1958 J. Am. Chem. Soc. 802639

13. Perrin D D, Armarego W L and Perrin D R 1966 Purification of organic compunds (Oxford: Pergamon)

14. Bradfield A E, Jones B and Orton K J P 1929 J. Chem. Soc. 2810

15. Exner O 1973 Prog. Phys. Org. Chem. 10411

16. Banerji K K 1973 Indian J. Chem. 11244

17. Falnberg A H and Winstein S 1956 J. Am. Chem. Soc. 782770

18. Wiberg K B 1963 Physical organic chemistry (New York: Wiley) 414

19. Exner O 1964 Collect. Czech. Chem. Commun. 291094

20. Pavelich W A and Taft R W 1957 J. Am. Chem. Soc. 794935

21. Mathur D, Sharma P K and Banerji K K 1993 J. Chem. Soc., Perkin Trans. 2205

22. Gould E S 1964 Mechanism and structure in organic chemistry (New York: Holt, Rinehart and Winston) p. 181 